\title{
Direct Observation of Plasma Waves and Dynamics Induced by Laser-Accelerated Electron Beams
}

\author{
M. F. Gilljohann, ${ }^{1,2}$ H. Ding, ${ }^{1,2}$ A. Döpp, ${ }^{1,2, *}$ J. Götzfried, ${ }^{1}$ S. Schindler, ${ }^{1}$ G. Schilling, ${ }^{1}$ S. Corde, ${ }^{3}$ A. Debus, ${ }^{4}$ \\ T. Heinemann, ${ }^{5,6}$ B. Hidding, ${ }^{5,7}$ S. M. Hooker, ${ }^{8}$ A. Irman, ${ }^{4}$ O. Kononenko, ${ }^{3}$ T. Kurz, ${ }^{4}$ \\ A. Martinez de la Ossa, ${ }^{6}$ U. Schramm, ${ }^{4}$ and S. Karsch ${ }^{1,2, \dagger}$ \\ ${ }^{1}$ Ludwig-Maximilians-Universität München, Am Coulombwall 1, 85748 Garching, Germany \\ ${ }^{2}$ Max Planck Institut für Quantenoptik, Hans-Kopfermann-Strasse 1, Garching 85748, Germany \\ ${ }^{3}$ LOA, ENSTA ParisTech-CNRS-École Polytechnique-Université Paris-Saclay, \\ 828 Boulevard des Maréchaux, 91762 Palaiseau Cedex, France \\ ${ }^{4}$ Helmholtz-Zentrum Dresden-Rossendorf, Institute of Radiation Physics, \\ Bautzner Landstrasse 400, 01328 Dresden, Germany \\ ${ }^{5}$ Scottish Universities Physics Alliance, Department of Physics, University of Strathclyde, \\ Glasgow G4 ONG, United Kingdom \\ ${ }^{6}$ Deutsches Elektronen-Synchrotron DESY, D-22607 Hamburg, Germany \\ ${ }^{7}$ Cockcroft Institute, Sci-Tech Daresbury, Keckwick Lane, Daresbury, \\ Cheshire WA4 4AD, United Kingdom \\ ${ }^{8}$ John Adams Institute \& Department of Physics, Clarendon Laboratory, University of Oxford, \\ Parks Road, Oxford OX1 3PU, United Kingdom
}

(Received 26 October 2018; published 12 March 2019)

\begin{abstract}
Plasma wakefield acceleration (PWFA) is a novel acceleration technique with promising prospects for both particle colliders and light sources. However, PWFA research has so far been limited to a few largescale accelerator facilities worldwide. Here, we present first results on plasma wakefield generation using electron beams accelerated with a 100-TW-class Ti:sapphire laser. Because of their ultrashort duration and high charge density, the laser-accelerated electron bunches are suitable to drive plasma waves at electron densities in the order of $10^{19} \mathrm{~cm}^{-3}$. We capture the beam-induced plasma dynamics with femtosecond resolution using few-cycle optical probing and, in addition to the plasma wave itself, we observe a distinctive transverse ion motion in its trail. This previously unobserved phenomenon can be explained by the ponderomotive force of the plasma wave acting on the ions, resulting in a modulation of the plasma density over many picoseconds. Because of the scaling laws of plasma wakefield generation, results obtained at high plasma density using high-current laser-accelerated electron beams can be readily scaled to low-density systems. Laser-driven PWFA experiments can thus act as miniature models for their larger, conventional counterparts. Furthermore, our results pave the way towards a novel generation of laser-driven PWFA, which can potentially provide ultralow emittance beams within a compact setup.
\end{abstract}

DOI: 10.1103/PhysRevX.9.011046

\section{INTRODUCTION}

Over the past century, particle accelerators and colliders have been an essential tool to discover new physics. Electron accelerators based on radio frequency (rf) technology have pushed the frontier of high-energy physics to the $100 \mathrm{GeV}$ level. However, to reach the tera-electron-volt

\footnotetext{
*andreas.doepp@physik.uni-muenchen.de

'stefan.karsch@physik.uni-muenchen.de
}

Published by the American Physical Society under the terms of the Creative Commons Attribution 4.0 International license. Further distribution of this work must maintain attribution to the author(s) and the published article's title, journal citation, and DOI.
Subject Areas: Photonics, Plasma Physics

frontier, the limited acceleration gradient $(\lesssim 100 \mathrm{MV} / \mathrm{m})$ of rf technology means that tens of kilometers of acceleration length are required and such accelerators will eventually become too expensive to be built [1]. Accordingly, a number of alternative accelerator concepts have been explored over the last decades. One of the most promising is wakefield acceleration in plasmas [2], which relies on an intense particle or laser beam to excite a relativistic plasma wave with field strengths exceeding hundreds of gigavolts per meter [3].

The concept of beam-driven plasma wakefield acceleration (PWFA) was developed in the 1980s [4,5]. First experiments showing modest acceleration and the onset of self-focusing were performed shortly later at the Argonne National Laboratory [6,7]. A major breakthrough was the 
observation of energy doubling of a $42 \mathrm{GeV}$ electron beam in an 85-cm-long PWFA at SLAC, which was reported in 2007 [8]. More recent experiments also demonstrated an energy transfer efficiency exceeding 30\% [9], first highenergy positron acceleration [10] and $\mathrm{GeV}$ electron acceleration using proton-driven PWFA [11]. In the future, advanced injection methods are expected to provide ultralow emittance electron beams [12-16], e.g., for compact free-electron lasers [17], and proton-driven PWFA has the potential to accelerate electron beams to tera-electron-voltscale energies [18].

An important parameter to characterize electron-beam drivers for PWFA is the peak charge density of the bunch, which is given by

$$
\rho_{b}=-e n_{b}=\frac{Q}{(2 \pi)^{3 / 2} \sigma_{z} \sigma_{r}^{2}}=\frac{I}{2 \pi c \sigma_{r}^{2}}
$$

for a Gaussian beam. Here, $e$ is the elementary charge, $n_{b}$ the peak particle density, $Q$ denotes the beam charge, $I$ is the peak current, $\sigma_{r}$ is the root mean square (rms) transverse beam size, and $\sigma_{z}$ is the rms bunch length. To exploit the multi-gigavolt-per-meter field gradients offered by the generation of nonlinear wakefields, $n_{b}$ needs to be on the order of the plasma density $n_{0}$. In addition, the temporal bunch profile should be matched to the plasma wavelength

$$
\lambda_{p}=2 \pi c \sqrt{\frac{\epsilon_{0} m_{e}}{e^{2} n_{0}}} \approx 1 \mathrm{~mm} \times \sqrt{\frac{1}{n_{0}\left[10^{15} \mathrm{~cm}^{-3}\right]}},
$$

with $c$ the speed of light, $\epsilon_{0}$ the vacuum permittivity, $m_{e}$ the electron mass, and $e$ the elementary charge.

The maximum accelerating field of a wakefield accelerator can be estimated by the cold wave-breaking field [19]

$$
E_{0}=\frac{2 \pi m_{e} c^{2}}{e \lambda_{p}} \approx 3 \mathrm{GV} \mathrm{m}^{-1} \times \sqrt{n_{0}\left[10^{15} \mathrm{~cm}^{-3}\right]}
$$

and, accordingly, a PWFA needs to be operated at densities $\gtrsim 10^{12} \mathrm{~cm}^{-3}$ in order to generate higher accelerating fields than common rf accelerators. But at the same time, meeting the above requirements to drive a wakefield becomes more challenging at higher plasma densities and currently only very few large-scale facilities worldwide are suitable to study PWFA and related plasma physics [20,21], typically at densities $n_{0} \sim 10^{14}-10^{17} \mathrm{~cm}^{-3}$. Hence, numerical studies are often used to provide insight into the physics of PWFA, but the combination of micrometer plasma dynamics with meter-scale acceleration lengths requires simplified geometries and models to limit the computational costs [22].

Here, we discuss a new experimental approach to study PWFA by using laser-wakefield-accelerated (LWFA) $[19,23,24]$ electrons as a plasma-wave driver [25,26].
Because of their unprecedented peak currents and few-fs duration [27], they allow the study of PWFA on much shorter spatial and temporal scales, corresponding to plasma densities in the $10^{18}-10^{20} \mathrm{~cm}^{-3}$ regime and field gradients approaching $100 \mathrm{GV} / \mathrm{m}$, with commercially available 100 TW-class Ti:sapphire lasers as the primary driver.

As the physics of PWFA is completely scalable with the plasma density, depending only on the relative bunch density $n_{b} / n_{0}$ and size $k_{p} \sigma_{z \mid r}$ (with $k_{p}=2 \pi / \lambda_{p}$ ), a LWFAdriven high-density PWFA can serve as a miniature model for large plasma accelerators such as FACET at SLAC [28], FLASHForward at DESY [29], or AWAKE at CERN [30]. It can thus provide a compact way to study physics related to beam-driven wakefield generation.

Beside its compactness, laser-driven PWFA offers several other advantages to its rf-driven counterparts. First, as they can be operated at densities exceeding $10^{18} \mathrm{~cm}^{-3}$, it is possible to use shadowgraphy with few-cycle optical probes [31,32] to study the interaction [33]. As these probes are usually derived from the same laser system, they are inherently synchronized to the laser-accelerated electron beam and can therefore provide snapshots of the plasma evolution with femtosecond jitter. Also, synchronized laser pulses can be used to provide accurately timed witness bunches, i.e., by techniques such as Trojan-horse injection [12] for the production of low-emittance beams, or dual-energy electron beams [34] with variable delay as driver-witness beams for probing the wakefield. Even the relatively large energy spread of the electron bunches typically generated by LWFA is beneficial for driving PWFA, because it suppresses beam hosing [35].

So far, only indirect signs for a transition from LWFA to PWFA have been observed, based on either electron energy measurements [36,37], pulse duration measurements [38], or x-ray emission diagnostics [39]. First experiments dedicated to PWFA with laser-accelerated electron beams observed an electron deceleration signature [40] and electron-beam focusing [41] in a second gas target. Here, we present the first direct and unambiguous observation of a plasma wave driven by laseraccelerated electrons using few-cycle shadowgraphy [31]. Furthermore, we present novel results on picosecondtimescale plasma ion dynamics behind the laser-generated electron-beam driver, which demonstrate the capabilities of laser systems to advance PWFA research.

\section{EXPERIMENTAL METHODS}

\section{A. Laser system}

The experiments were performed with the ATLAS laser at the Laboratory for Extreme Photonics, Garching. During the experiments, the Ti:sapphire chirped pulse amplification system delivered 800-nm central wavelength laser pulses of 28-fs duration and 2.5-J energy on target, corresponding to a peak power of $84 \mathrm{TW}$. 


\section{B. Few-cycle shadowgraphy}

To obtain few-cycle probe pulses suitable for the shadowgraphy of plasma waves, a small part of the laser pulse (about $1 \mathrm{~mJ}$ ), is coupled out before the focusing optics and sent into an argon-filled hollow-core fiber. Selfphase modulation (SPM) inside the fiber leads to spectral broadening and allows temporal compression of the beam to below $10 \mathrm{fs}$, while its timing is adjusted with a delay stage (see the Appendix for more details). It is sent through the target perpendicularly to the main pulse. The plane of interaction is imaged by a long-working-distance microscope objective $(5 \times$ or $10 \times$ magnification, depending on the configuration) to form shadowgrams with a spatial resolution of approximately $2 \mu \mathrm{m}$. Because of the short pulse duration even rapidly moving structures like plasma waves can be resolved. The measured diffraction signal directly reflects periodic modulations of the plasma density distribution, i.e., the laser- or beam-driven plasma wave. In the quasilinear regime of wakefield acceleration, the periodicity of the plasma-wave train is equal to the plasma wavelength $\lambda_{p}$, which is 10 to $30 \mu \mathrm{m}$ for densities $n_{0}$ of $10^{19}$ and $10^{18} \mathrm{~cm}^{-3}$, respectively [cf. Eq. (2)]. Meanwhile, the transverse size of the shadowgram depends not only on the wave's diameter, but also on the distance between plasma wave and the image plane, which is not precisely known due to the drive pulse's pointing jitter. By adding an optional Wollaston prism and a polarizer, the probe beam can also be used to implement an in situ Nomarski-type interferometer to characterize the density of the plasma channel created by the drive beam.

\section{Target configuration}

A 3D rendering of the setup in the vacuum chamber is shown in Fig. 1. For laser wakefield acceleration, the horizontally polarized laser pulses are focused into a supersonic hydrogen gas jet target (hereafter referred to as the first jet) using a $f / 25$ off-axis parabola, reaching an estimated peak normalized vector potential $a_{0}=$ $e A_{0} / m_{e} c^{2}=1.6$ at focus. A subsequent hydrogen gas jet (hereafter referred to as the second jet) was installed downstream of the first jet, at variable distance and with independent flow control. Optionally, a part of the main beam could be coupled out before the final focusing optics via a pick-off mirror and delay stage to provide an

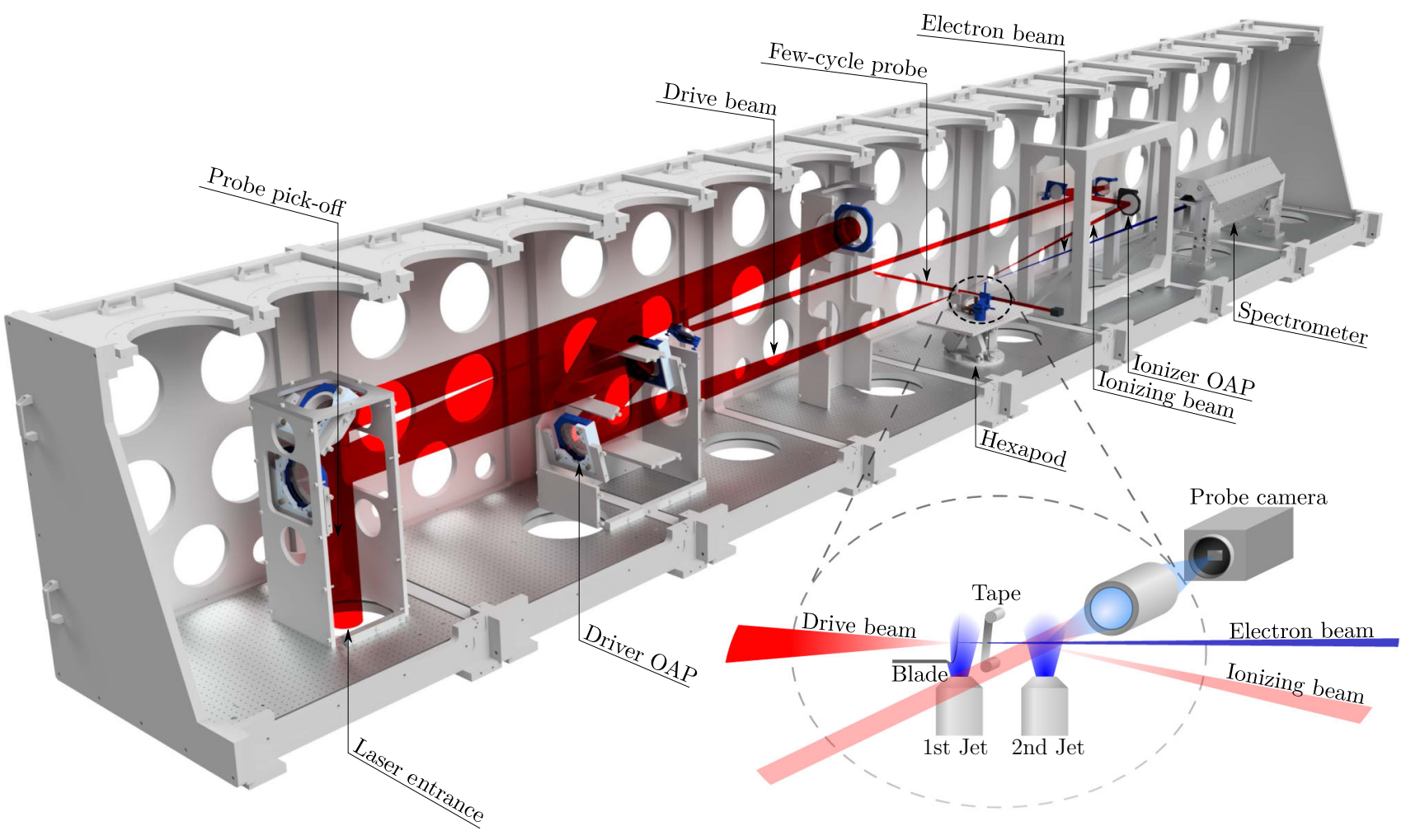

FIG. 1. Experimental setup. The probe pulse is picked from the main beam at the chamber entrance and is coupled into a SPM-based broadening and compression setup outside of the vacuum chamber (not shown here). Meanwhile, the drive beam ( $2 \mathrm{~J}, 30 \mathrm{fs}$ ) is delayed by about $20 \mathrm{~ns}$ to accommodate for the additional delay of the few-cycle probe. The gas target and probe imaging setup are mounted on a hexapod stage in focus of the off-axis parabola (OAP). The profile of the laser-accelerated electron beam (indicated in blue) is measured with a scintillating screen (not shown here) mounted in front of the dipole magnet spectrometer. The ionizing pulse (about $60 \mathrm{~mJ}$ ) is also picked from the main beam and focused using a second OAP at an angle of $173^{\circ}$ to the drive beam. Bottom right: Larger sketch of the target geometry, showing the two gas jets, the optional tape drive to block the laser, and the three laser beams. 
independently timed counterpropagating laser pulse (similar to Ref. [42]) to ionize the second jet.

\section{Laser wakefield accelerator}

As a first jet, supersonic gas nozzles with 3- and 5-mm diameter were used. To facilitate electron injection, a silicon wafer was moved into the gas stream, leading to the formation of a shock front [43-45]. The jet was operated in a density range of $3 \times 10^{18} \mathrm{~cm}^{-3}$ to $6 \times 10^{18} \mathrm{~cm}^{-3}$, which was in each specific configuration close to the threshold for self-injection. Shock-front injectors are usually operated at densities well below this threshold to generate monochromatic electron beams. Increasing the density leads to a higher energy spread but also substantially higher injected charge. This resulted in beams with up to $900 \mathrm{pC}$ in the energy range of $25-400 \mathrm{MeV}$ at $150 \mathrm{MeV}$ central energy and down to $0.6 \mathrm{mrad}$ FWHM divergence (see the Appendix for representative electron spectra and Ref. [46] for details on the charge calibration). While the pulse duration is not directly measured in this experiment, previous bunch-length measurements $[27,38]$ suggest a duration of about $5 \mathrm{fs}$, corresponding to peak currents of up to $170 \mathrm{kA}$.

\section{RESULTS}

Here, we present the results of three experiments, each with a different configuration.

In the first setup we observe two plasma waves in the second jet (see Fig. 2), one of which has a distinct conelike diffraction feature which we never observed for laser-driven plasma waves. This leads to the assumption that this wave is driven by the electron beam from the first jet. To verify this hypothesis, we block the laser with a tape in the second experimental configuration. When we preionize the gas in the second jet we observed an unequivocally beam-driven plasma wave. It is accompanied by the same conelike diffraction feature as the supposed beam-driven wave in the first experiment (see Fig. 3). In a third experiment we study this cone feature (see Fig. 4), which turns out to be caused by the ion motion of beam-driven plasma waves.

A summary of the target parameters in each experiment can be found in Table I in the Appendix.

\section{A. Observation of two plasma waves in a second gas target}

During LWFA, the electron beam is confined to the vicinity of the optical axis due to the transverse electrostatic wakefield [49]. In this situation, the electron beam does not drive its own wave, but only affects the laser-driven wave via beam loading [50] until the laser depletes or the electron beam overtakes the laser. In both cases the laser will still perturb the beam-driven wave to a degree that is difficult to measure or predict. In order to observe a purely beamdriven wave, one therefore needs to isolate the electron beam, i.e., by blocking the laser with a foil [40]. However, scattering in the foil increases the electron bunch emittance and radius $\sigma_{r}$ after further propagation, which reduces its peak density $n_{b} \propto \sigma_{r}^{-2}$.

As an alternative, we exploit the fact that the electron-beam pointing is not necessarily collinear to the laser axis. For instance, a slight pulse-front tilt of the laser pulse can lead to skewed plasma-wave fronts [51]. Hence, the laser and electron beam propagate at different angles in the space between both jets, leading to a spatial separation. In this first experiment we generate a beam with $200 \mathrm{pC}$ (about $40 \mathrm{kA}$ ), $0.6 \mathrm{mrad}$ FWHM divergence, and a mean energy of $150 \mathrm{MeV}$ in the first jet. Indeed, as shown in Fig. 2, for most shots we observe two distinct plasma waves in the second jet, which is placed after a 3-mm vacuum gap behind the first jet. For the upper plasma wave we measure a wavelength of $(7.6 \pm 0.1) \mu \mathrm{m}$, for the lower one $(7.8 \pm 0.1) \mu \mathrm{m}$. The difference of $2.6 \%$ can be caused either by a weak nonlinearity or a local difference of the plasma density $n_{0}=(1.9 \pm 0.1) \times 10^{19} \mathrm{~cm}^{-3}$. Accordingly, any laser contribution is expected to be weak, with a peak potential $a_{0} \lesssim 1$.

In principle, it cannot be ruled out a priori that both of these waves are driven by laser filaments. However, a

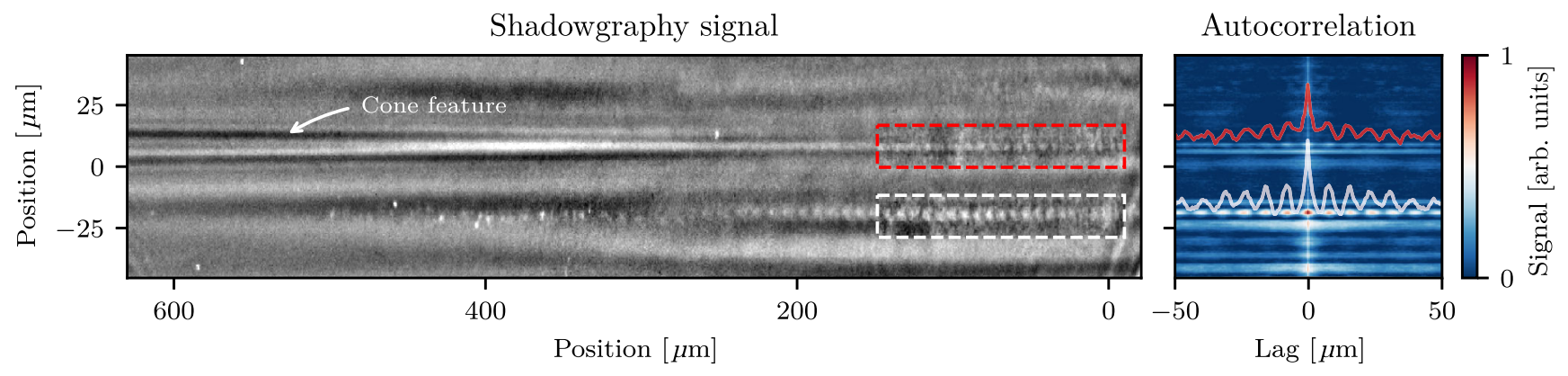

FIG. 2. Shadowgram of laser- and beam-driven plasma waves in the second gas jet. Left: Laser- and beam-driven plasma waves in the second gas jet (propagating to the right) after a free drift and spatial separation. Note the conelike feature trailing only the upper plasma wave. Right: Autocorrelation of each row of the signal in the interval of the marked plasma waves on the left. The red and white lineouts show the respective periodic signal modulations caused by the plasma waves. 
marked difference in the morphology of both signals is the conelike structure trailing one of the plasma waves. As will be discussed later, this feature is attributed to the dynamics of background plasma ions and a signature for electrondriven waves.

\section{B. Observation of purely beam-driven plasma waves}

To verify that one of the plasma waves is really driven by an electron beam, we perform the second experiment, where the setup is changed, such that the laser is blocked between the gas jets with a $15-\mu$ m-thick Mylar tape acting as a plasma mirror $[52,53]$. As mentioned before, the foil defocuses the electron beam. In our measurements the divergence increases by a factor $\alpha=2.7 \pm 1.5$, which results in a decrease of the wave amplitude by a factor of up to $\alpha^{-2}$. In order to minimize the increase in beam size, the first jet and the tape need to be as close to the second jet as possible. Because of geometrical constraints in our setup, the minimal distance between the jets was $10 \mathrm{~mm}$ and the distance from the first jet to the tape was $2 \mathrm{~mm}$.

In this configuration, the LWFA produces 900-pC electron beams. Figure 3(a1) shows that this bunch causes a transverse diffraction pattern in the shadowgram of the second jet, which indicates that the neutral gas is at least weakly ionized by the electron beam. However, there is no visible sign of a plasma wave and the autocorrelation of the data [Fig. 3(b1)] shows no obvious periodic features in longitudinal direction indicative of a plasma wave. This is likely the result of the missing preionization by the laser and the fact that the foil-induced defocusing prevents the beam from becoming dense enough for causing more than weak ionization. Ionization occurs only when the transverse electrostatic fields of the bunch exceed the field ionization threshold, which is about $25 \mathrm{GV} \mathrm{m}^{-1}$ for an
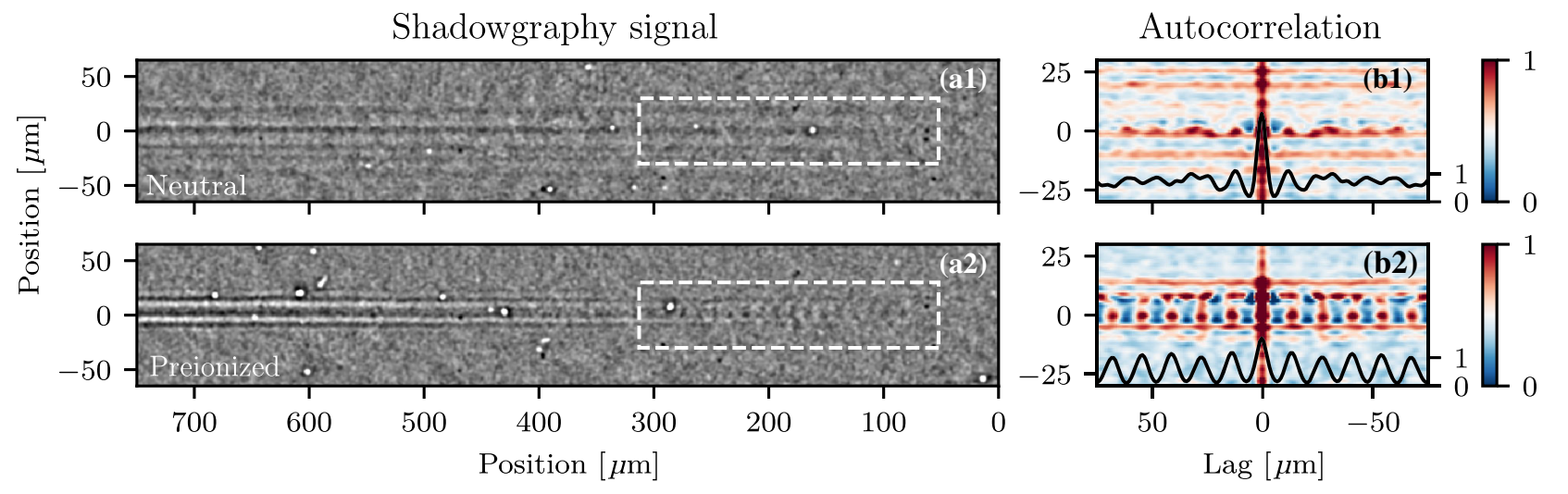

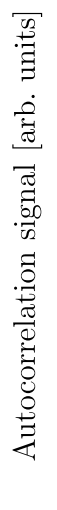
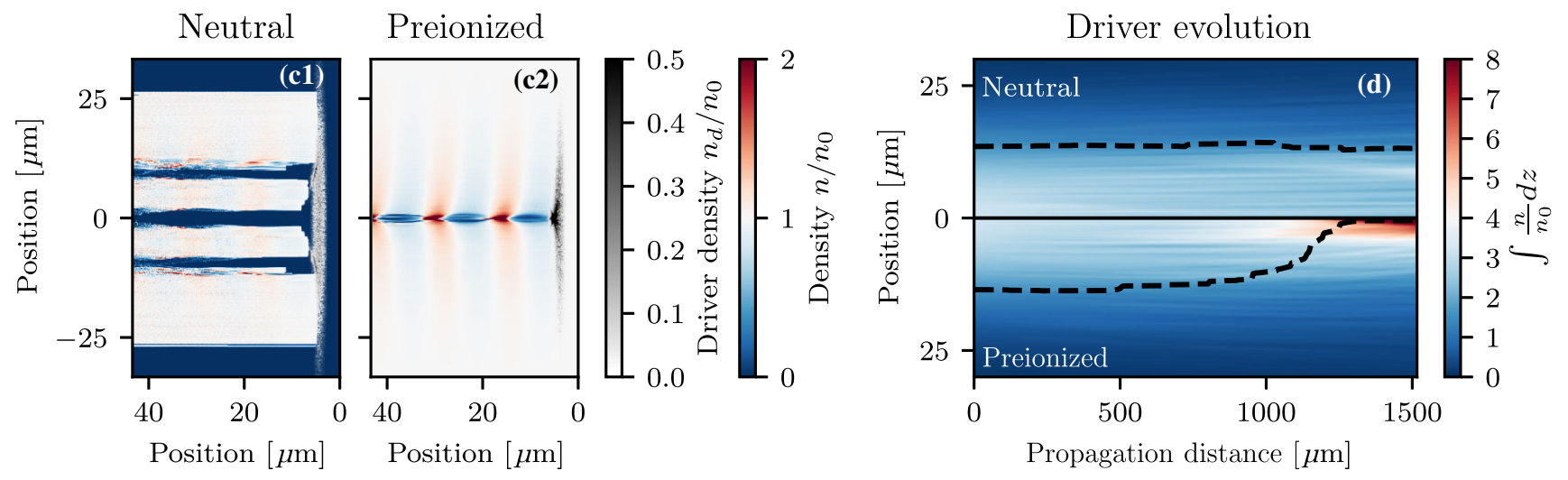

FIG. 3. Electron-driven plasma waves with blocked laser. Top: (a) Shadowgrams of the second jet for neutral and preionized hydrogen gas. The drive bunch propagates from left to right. (b) Row-wise autocorrelations within the region of interest (dashed rectangle). The graphs in (b) are the horizontal lineouts at vertical zero. The transverse modulation in the neutral case can only be attributed to ionization from the electron beam. The autocorrelation (b1) shows no indication of a longitudinal signal modulation that would be generated by a plasma wave. However, the preionized case (a2) shows a weak, but visible periodical longitudinal modulation which is caused by a plasma wave driven by the electron beam. This modulation is clearly visible in the autocorrelation (b2). Bottom: Full 3D simulations of the interaction. (c) Charge densities of the background plasma electrons (blue-red color scale) along with the driver (gray color scale). The regions in (c1) where the electron density is zero correspond to nonionized gas. (d) Evolution of the transverse (longitudinally integrated) driver density for both cases, along with their half width at half maximum (HWHM, dashed line). The driver in the preionized case self-focuses much faster and drives a stronger plasma wave, even with full blowout of the background electrons. In the neutral case the driver is not able to fully ionize the gas. See Fig. S3 in the Supplemental Material [47] for a close-up of the drivers. 
ionization probability of $1 \%$ per fs in atomic hydrogen [54]. Hence, the head of the bunch, in front of the ionization, does not contribute to the wave generation. Furthermore, in the radial direction, the fields are zero in the center and reach a maximum at $\sigma_{r}$, which leads to an annular-shaped ionization trace.

To overcome this problem, a counterpropagating pulse is used to preionize the gas several picoseconds before the arrival of the electron beam. The ionization pulse has an energy of about $60 \mathrm{~mJ}$ and intercepts the electron bunch at an angle of $173^{\circ}$ to the driver axis.

In this case, the shadowgram in Fig. 3(a2) along with the autocorrelation [Fig. 3(b2)] shows a periodical longitudinal modulation at the plasma wavelength. Since the laser driver from the first jet is blocked by the tape, this unequivocally is an isolated, purely electron-driven plasma wave. We measure a plasma wavelength of $(13.6 \pm 0.3) \mu \mathrm{m}$, which is in accordance with the plasma wavelength of $(13.2 \pm$ $0.2) \mu \mathrm{m}$ [equivalent to $n_{0}=(6.2 \pm 0.2) \times 10^{18} \mathrm{~cm}^{-3}$ ] from measurements without tape in otherwise identical conditions. Note that the shadowgram shows a similar diffraction feature as observed behind one of the plasma waves in Fig. 2.

To verify our interpretation of the results, we perform full-3D particle-in-cell (PIC) simulations using OSIRIS 4.4 [55], with and without preionization. The bunch exiting the first jet was measured to contain a total charge of $900 \mathrm{pC}$, of which $550 \mathrm{pC}$ were transmitted through the second jet (see Fig. 5 in the Appendix). Half of the spectrum was detected in a low-energy (and/or highly divergent) background, which is unlikely to contribute significantly to the plasma-wave generation. Thus, for the simulations only the bunch charge between 100 to $350 \mathrm{MeV}$ was considered, which amounts to $300 \mathrm{pC}$. The transverse size was calculated to $\sigma_{r}=11.8 \mu \mathrm{m}$ from the average measured divergence. The spatially correlated momenta in the simulations were initialized according to the free drift with a divergence of $1.7 \mathrm{mrad}$ and a temperature of $40 \mathrm{keV}$. The temporal length was assumed to be 5 fs FWHM, which corresponds to a peak current of $56 \mathrm{kA}$. The moving simulation box has a size of $(x \times y \times z)=(60 \times 60 \times 20) k_{p}^{-3}$ at a resolution of $\Delta x=\Delta y=\Delta z=0.05 k_{p}^{-1} \simeq 0.1 \mu \mathrm{m}$ (with $n_{0}=6.4 \times 10^{18} \mathrm{~cm}^{-3}$ ), and each cell is initialized with one electron macroparticle. For simulations with an initially neutral gas, OSIRIS employs a field ionization model [54] to calculate ionization probabilities.

The simulation results are shown in Figs. 3(c) and 3(d). We observe that the driver alone is not able to ionize the gas over its full extent [Fig. 3(c1)] and self-focuses much less than in the preionized case [see Fig. 3(c2) and Fig. S3 in the Supplemental Material [47] for close-ups of the drivers]. More specifically, it evolves into a funnel-like shape and ionizes two rings, which resembles the observed diffraction structure in Fig. 3(a1). In contrast, in the preionized case, the driver self-focuses much more strongly and in turn drives a higher amplitude plasma wave. The simulation also predicts that the tail can drive a few-micron-radius plasma wave in full blowout of the background electrons. However, such a small region would induce a weak phase shift compared to the larger linear plasma wave and is therefore not observable in the shadowgraphy.

To conclude, in this experiment we have observed beamdriven plasma waves at densities of about $10^{19} \mathrm{~cm}^{-3}$ for the first time and, due to the tape, we can rule out any influence of the laser.

\section{Observation of ponderomotive ion channel formation}

Beside the observation of a periodic intensity modulation from to the plasma wave, the shadowgrams also frequently show an unexpected, conelike feature. So far, we have observed that this feature formed in most cases in the second jet without foil and always with foil [see Fig. 3(a2) and Supplemental Material, Figs. S1 and S2 [47], for a full field of view]. In contrast, the feature is not present in any of the first-jet LWFA shadowgrams that we acquired and it is also absent in the second jet when there is no electron beam generated in the first jet. Therefore, we can conclude that the cone is indeed a distinguishing feature of electron-driven plasma waves, at least for our experimental conditions.

To further investigate this effect, we perform a third experiment that concentrates on the features of the cone. The large field of view of the shadowgraphy diagnostic allows us to study the evolution on a picosecond timescale. In order to spoil the electron driver as little as possible, we remove the tape and move the jets closer to each other. The configuration is similar to the first experiment, but with slightly increased separation and almost twice the density in the first jet (cf. Table I). This leads to more than $2.5 \times$ the beam charge $(520 \mathrm{pC}$, about $100 \mathrm{kA})$ and less transmitted laser energy. Accordingly, we observe only one plasma wave, always accompanied by a cone. As shown in Fig. 4(a) and figures in the Supplemental Material [47], its origin is located close to the tail of the plasma wave, starting after a few hundred femtoseconds, and it persists at least out to $50 \mathrm{ps}$, as confirmed by varying the probe pulse delay. We measure a half-opening angle $\alpha=$ $(3.0 \pm 0.5) \mathrm{mrad}$ of the cone in this specific configuration.

To our knowledge, no similar observation has been reported for either LWFA or PWFA and the origin of the diffraction cone was initially unclear. Assuming a mostly perpendicular motion, a transverse (group) velocity of $v_{\perp}=0.0017 \mathrm{c}$ can be inferred from the opening angle. If the ion background was static and this feature arose only from electron motion, the velocities would be far too low to sustain a charge separation and the restoring forces would lead to plasma oscillations. Yet, the latter are not observed and the feature has to be associated with ion motion.

We therefore perform PIC simulations with a mobile ion background. In order to cover the several-picosecond-long 

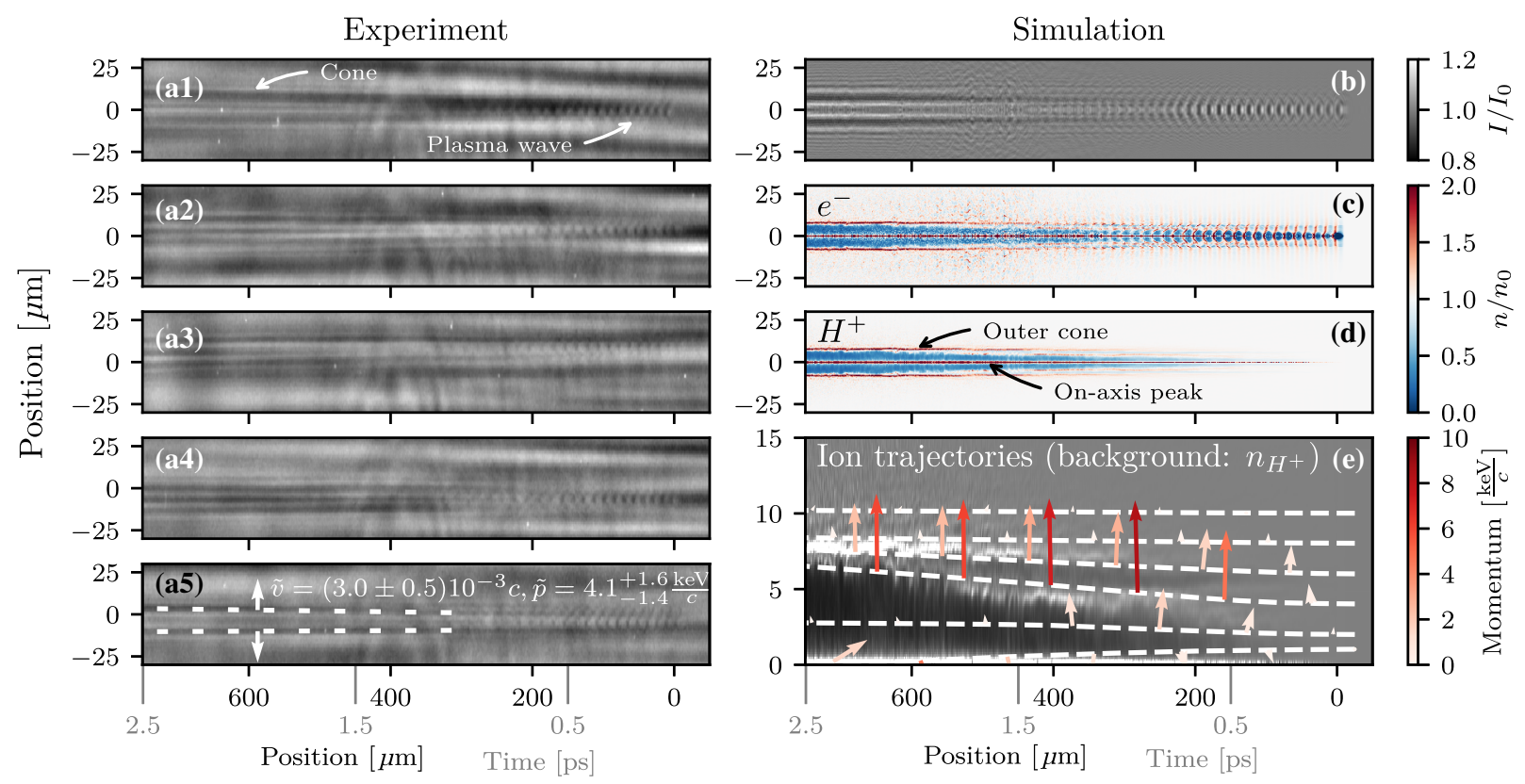

FIG. 4. Ion-channel formation from a plasma wakefield. Left: (a) Raw shadowgrams showing electron-driven plasma waves (propagating to the right) and their trailing ion channels for five consecutive shots. The dashed lines in the lower shadowgram exemplarily show the maxima of the ion distribution (via the electron distribution), the radial velocity of the maxima $\tilde{v}$ and the momentum of an ion with $\tilde{p}=m_{i} \tilde{v}$. Right: Corresponding particle-in-cell simulations and synthetic shadowgram (b). The electron (c) and ion densities (d) clearly show quasineutrality after several plasma-wave periods. The channel in the synthetic shadowgram is in excellent agreement with the measured ones. The ion trajectories (e) on a radially scaled ion density from (d) show that ions close to the symmetry axis are accelerated towards the axis, while ions with $r_{0} \gtrsim 2 k_{p}^{-1}$ are accelerated away from it. Arrows along with the color scale indicate the instantaneous momenta.

experimental observation window, we assume a symmetric beam driver and perform simulations in cylindrical coordinates. The drive-bunch parameters are deduced from reference shots with the second jet switched off, i.e., $520 \mathrm{pC}$ at $150 \mathrm{MeV}$ and $14-\mu \mathrm{m}$ width at the second jet. The simulation window has a size of $(r \times z)=(45 \times 440) k_{p}^{-2}$, at a resolution of $\Delta r=\Delta z=0.033 k_{p}^{-1}$, with $n_{0}=6 \times 10^{18} \mathrm{~cm}^{-3}$ inferred from interferometry measurements. In each cell of the mesh, four electron and four ion macroparticles are initialized.

The simulations [see Figs. 4(b)-4(e)] indeed show a conelike structure appearing in the ion distribution in the trail of the wake. While our shadowgraphy diagnostic is sensitive to diffraction caused by changes in the local electron density, the ion distribution itself is not visible. However, the plasma-wave decays after around $400 \mu \mathrm{m}$ behind the driver such that the large charge imbalance vanishes and the plasma becomes quasineutral, leading to approximately equal electron and ion distributions from 400 to $700 \mu \mathrm{m}$. As a result, also the electron distribution exhibits the cone-shaped structure, which allows us to observe this ion motion using shadowgraphy.

For better comparison with the experimental data, we simulate the propagation of the probe through the electron distribution calculated in the PIC simulation (see the Appendix for more information). The synthetic shadowgram, shown in Fig. 4(b), is in excellent agreement with the experimental data and reproduces the same diffraction features. The radial velocity of the ion momentum $m_{i} v_{\perp}^{\text {sim }} \sim 4 \mathrm{keV} / \mathrm{c}$ is also compatible with the measured $m_{i} v_{\perp}^{\exp }=4.1_{-1.4}^{+1.6} \mathrm{keV} / c$.

However, our analysis shows that the mechanism causing the ion motion differs from common ion channel formation due to Coulomb explosion [56,57]. While a Coulomb explosion leads to a radial expulsion of ions, and, hence, an annularly shaped distribution, the ion density in our simulations also increases close to the propagation axis. The reason for this is that the ions in a plasma wave experience radial focusing and defocusing fields in alternation. The net effect of such oscillating forces can be calculated using the ponderomotive formalism. In the nonrelativistic limit, which is justified since $v_{\perp}=$ $0.0017 \mathrm{c} \ll c$, the ponderomotive force exerted by the plasma wave is [58]

$$
\vec{F}_{\text {pond,PW }}=-\frac{e^{2}}{4 \omega_{p}^{2}} \vec{\nabla}\left|\vec{E}_{\mathrm{PW}}\right|^{2}
$$

where $\vec{E}_{\mathrm{PW}}$ is the local amplitude vector of the wakefield. In contrast to the well-known ponderomotive force of a laser pulse, the plasma-wave amplitude remains almost constant 
over many periods (equivalent to a flat envelope) so the ponderomotive force of the plasma wave acts mainly radially. Since the radial electric fields of a plasma wave vanish on axis, the intensity gradient points towards $r \rightarrow 0$ for ions close to the symmetry axis, which results in the formation of a density peak on axis and an annular region of ions expanding outwards, as visualized in Fig. 4(e). This effect was predicted in analytical and numerical studies of laser-driven waves by Gorbunov et al. [60,61], for intense electron-beam drivers by Rosenzweig et al. [62] and for self-modulated plasma wakefield accelerators by Vieira et al. [63,64].

However, despite the prediction of a similar ponderomotive ion motion for laser-driven plasma waves, we only observed the diffraction pattern behind electron drivers. This observation can be explained by the different field gradients generated by both types of drivers. Electron bunches can self-focus to sizes of or below the skin depth [65], $\sigma_{r} \lesssim k_{p}^{-1}=\lambda_{p} / 2 \pi$, which leads to strong transverse gradients that in turn cause noticeable ion motion. In contrast, Gorbunov et al. [60] found that the depth and profile of the ion channel for laser-driven plasma waves depends to a large degree on the laser waist $w_{0}$. For laser waist sizes $w_{0} \gtrsim \lambda_{p} / 2=\pi k_{p}^{-1}$, the ion profile resembles a shallow on-axis depression channel, while for smaller laser waists the ion channel becomes deeper and the shape similar to the electron-driven case with a maximum on axis. Only the latter will lead to an electron distribution that can be detected using shadowgraphy, because the diffraction scales with the second derivative of the density. In our measurements the plasma wavelength in the first and second jet was $\lambda_{p}<19 \mu \mathrm{m}\left(n_{0} \gtrsim 3 \times 10^{18} \mathrm{~cm}^{-3}\right)$. Hence, the laser waist would need to be smaller than about $9.5 \mu \mathrm{m}$, which is well below both the Gaussian waist of $25 \mu \mathrm{m}$ and the matched spot size $w_{0}=2 \sqrt{a_{0}} k_{p}^{-1}$, explaining the missing diffraction feature for the laser-driven case.

We now concentrate on the motion of the outwards expanding ions. The kinetic energy of an ion tends towards the initial ponderomotive potential $\Phi_{\text {pond,PW }}=$ $\left(e^{2} / 4 m_{\text {ion }} \omega_{p}^{2}\right)\left|\vec{E}_{\mathrm{PW}}\right|^{2}$. Hence, the terminal velocity depends on the initial radial position $r_{0}$ of the ion, cf. momentum vectors in Fig. 4. Ions located further away from the wake's center will only experience a weak ponderomotive force and reach smaller velocities than ions with smaller initial radial position. Once the wake depletes and becomes quasineutral, the ions move mainly ballistically and the trajectories of ions with different velocities will cross. At this point, the amplitude of the transverse density modulation reaches its peak, which also results in a stronger Fresnel diffraction of the probe. However, most of the diffraction signal arises from the border between the lowdensity ring left behind by the ions and the high-density region. The expansion velocity of this ring, which will result in the conelike shape, is determined by the velocity of the innermost high-density region. Initially those are ions from the central region $\left(r_{0} \sim 3 k_{p}^{-1}\right)$, but once these ions overtake the slower ions with $r_{0} \gg 3 k_{p}^{-1}$, the cone's shape is determined by these slower ions. We observe this behavior in both experiment and simulations, where the initial opening angle just behind the plasma waves is larger than it is further behind the wakefield.

As mentioned in the introduction, one important feature of plasma wakefield formation is that it scales relative to the plasma parameters, i.e., with $n_{b} / n_{0}$ and $k_{p} \sigma_{z \mid r}$. Accordingly, most results are scalable to other plasma densities, time and length scales. The high current of laser-accelerated beams generally gives access to higher plasma densities than conventional accelerators. For instance, results from our laser-driven 1-mm-long PWFA operating at $10^{19} \mathrm{~cm}^{-3}$ can be scaled to a 10-cm-long PWFA operating at $10^{15} \mathrm{~cm}^{-3}$. Accordingly, our observation of the ion motion persisting up to $50 \mathrm{ps}$ implies that a PWFA with equivalent driver parameters at densities of $10^{15} \mathrm{~cm}^{-3}$ would observe ponderomotive ion motion on the timescale of nanoseconds.

The ion channel formation is an important energy dissipation channel in plasma wakefields, as energy is directly transferred from the plasma wave to the ion background. Furthermore, it has consequences especially for wakefield generation with long particle drivers like AWAKE, as the ion motion can lead to an early suppression of the self-modulation instability [35], and on PWFA with bunch trains where the plasma density might not be able to recover from the perturbation between two shots. Hence, our results on ion motion have immediate implications for the design of large, low-density PWFAs.

\section{CONCLUSIONS AND OUTLOOK}

We use laser-wakefield-accelerated electron beams, generated by a 100-TW-class laser, to study beam-driven plasma waves and dynamics. Our measurements unambiguously show that such electron beams can drive plasma waves at densities of about $10^{19} \mathrm{~cm}^{-3}$. We observe that preionizing the gas target is important in order to effectively drive a plasma wave with bunches having undergone emittance growth in a laser-blocking foil.

Importantly, the few-cycle shadowgraphy diagnostic not only gives access to femtosecond dynamics of the plasma wakefield, but also allows us to study the electron density evolution over the timescale of picoseconds in a single shot. In doing so, we observe a conelike diffraction pattern and simulations clearly attribute this feature to ion motion induced by the ponderomotive force of the beam-driven plasma wakefield. As the electron distribution follows the ion motion, the plasma density profile remains perturbed picoseconds behind the plasma wave. This feature is not observed for laser-driven plasma waves, which also allows us to distinguish laser- and beam-driven plasma waves in our experiment. 
Because of the physics of PWFA, results obtained at high plasma density using LWFA electrons can be immediately scaled to low-density scenarios relevant especially for large-scale future PWFA accelerators. The observed ion motion should, therefore, also occur at longer timescales at conventional PWFA facilities. Indeed, the same feature has been independently observed in recent experiments at the FACET user facility at SLAC [66]. This demonstrates that compact laser-driven setups can serve as a viable addition or even alternative to large-scale accelerator facilities in beam-driven plasma physics and accelerator research.

In the near future, petawatt laser systems such as the ATLAS-3000 laser in Garching or the Draco-PW laser in Dresden will be able to generate Joule-class $(\mathrm{nC} \times \mathrm{GeV})$ electron beams [67,68]. Using these systems, different regimes of beam-driven wakefield acceleration will be accessible using laboratory-scale systems, e.g., to produce scaled versions of meter-long PWFAs, bright $\gamma$-ray sources [69], or to generate highest-quality electron beams [12], with the latter having the potential to drive compact freeelectron lasers.

\section{ACKNOWLEDGMENTS}

The authors thank J. Vieira (IST) and M. Downer (U. Texas) for helpful discussions. This work was supported by DFG through the Cluster of Excellence MunichCentre for Advanced Photonics (MAP EXC 158) and SFB TR-18 funding schemes, by Euratom research and training programme under Grant agreement No. 633053 within the framework of the EUROfusion consortium, and the Max Planck Society. The authors gratefully acknowledge the Gauss Centre for Supercomputing e.V. for funding this project by providing computing time on the GCS Supercomputer SuperMUC at Leibniz Supercomputing Centre under Project No. pn69ri. The authors also would like to acknowledge the OSIRIS Consortium, consisting of University of California, Los Angeles (USA) and Instituto Superior Ténico (Lisbon, Portugal) for the use of OSIRIS and the visXD framework. S. C. and O. K. were supported by the European Research Council (ERC) under the European Unions Horizon 2020 research and innovation programme (Miniature beam- driven Plasma ACcelerators project, Grant Agreement No. 715807). S. M. H. was supported by a visitor grant from the Center for Advanced Studies (CAS) at LMU Munich.

M. F. G., H. D., A. D., J. G., S. S., G. S., S. M. H., and S. K. set up and/or performed the experiment. H. D. set up the few-cycle probe. M.F. G. analyzed the data and performed simulations. All authors discussed the results. M. F. G. and A. D. wrote the paper. S. K. supervised the project.

\section{APPENDIX: METHODOLOGY}

\section{Experiment configurations}

In this work, we present three experiments, each with a different target configuration.

All parameters of the respective setups are summarized in Table I. We define the respective entrance and exit of the gas jets with the position where the plasma starts becoming visible (corresponding to about $1 \times 10^{17} \mathrm{~cm}^{-3}$ ). The density ramps are 0.5 - to 1 -mm long, depending on the nozzle type and if a shock front is present. The separation between the jets is the length between the exit of the first and entrance of the second jet. The densities were determined with interferometric measurements and verified with the plasma wavelength from shadowgrams, and the uncertainty is found to be about $\pm 0.4 \times 10^{18} \mathrm{~cm}^{-3}$. Unless otherwise stated, these uncertainties apply.

\section{Electron beam spectra and beam profile}

Figure 5 shows representative electron-beam spectra and profiles from experiment 2. The 5-mm-long first jet with the shock-front injector was operated at a density of $2.9 \times 10^{18} \mathrm{~cm}^{-3}$. This resulted in beams with $900-\mathrm{pC}$ charge, spectra as representatively shown in Fig. 5 and $1.7 \mathrm{mrad}$ FWHM divergence. The beam charge was characterized using an absolutely calibrated scintillating screen; see Kurz et al. [46]. Note that in contrast to prior work, the shock-front injector was operated with optimized beam charge and divergence, which results in a broad

TABLE I. Configurations for Experiments 1, 2, and 3. The uncertainties of the densities are $\pm 0.4 \times 10^{18} \mathrm{~cm}^{-3}$. The charge is measured in the interval between 25 and $400 \mathrm{MeV}$.

\begin{tabular}{lccc}
\hline \hline & Experiment 1 & Experiment 2 & Experiment 3 \\
\hline Diameter of first jet & $3 \mathrm{~mm}$ & $5 \mathrm{~mm}$ & $3 \mathrm{~mm}$ \\
Density of first jet & $3.2 \times 10^{18} \mathrm{~cm}^{-3}$ & $2.9 \times 10^{18} \mathrm{~cm}^{-3}$ & $5.6 \times 10^{18} \mathrm{~cm}^{-3}$ \\
Charge from first jet & $200 \mathrm{pC}$ & $900 \mathrm{pC}$ & $520 \mathrm{pC}$ \\
Diameter of second jet & $1 \mathrm{~mm}$ & $3 \mathrm{~mm}$ & $1 \mathrm{~mm}$ \\
Density of second jet & $1.9 \times 10^{19} \mathrm{~cm}^{-3}$ & $6.0 \times 10^{18} \mathrm{~cm}^{-3}$ & $6.1 \times 10^{18} \mathrm{~cm}^{-3}$ \\
Jet separation & $3 \mathrm{~mm}$ & $10 \mathrm{~mm}$ & $3.5 \mathrm{~mm}$ \\
Tape & $\cdots$ & $15 \mu \mathrm{m} \mathrm{Mylar}$ & $\cdots$ \\
Separation tape to first jet & $\cdots$ & $2 \mathrm{~mm}$ & $\cdots$ \\
Ionizing beam & $\cdots$ & $60 \mathrm{~mJ}$ & $\cdots$ \\
\hline \hline
\end{tabular}



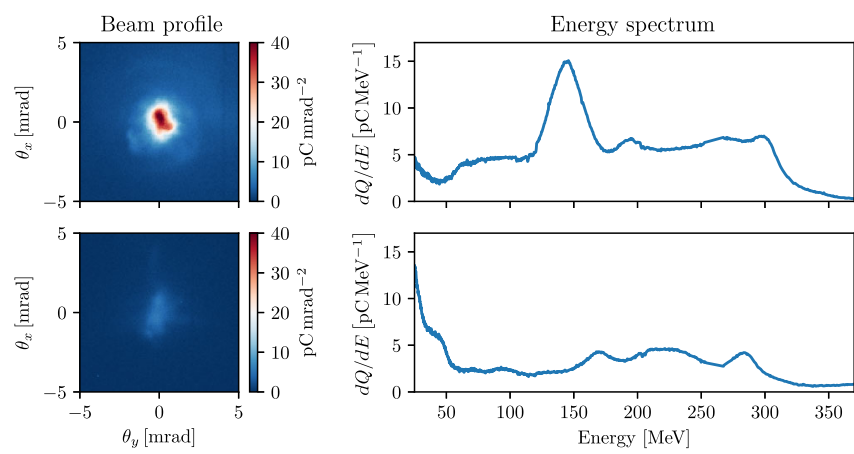

FIG. 5. Beam profile and energy spectra of representative shots with only the first gas jet (top) and with tape and second gas jet (bottom).

energy spectrum. Also, while the second jet clearly affects the spectrum and divergence of the electron beam, we do not observe any clear acceleration or deceleration effect. This is mainly due to the shot-to-shot fluctuations and the above-mentioned beam energy spread. The charge detected in the spectrometer decreased from 900 to $550 \mathrm{pC}$ when the tape was inserted and the second jet was activated, a reduction similar to Chou et al. [40].

\section{Few-cycle pulse generation}

The probe beam was derived from the main ATLAS beam using a half-inch mirror. This beam was then guided through a 1-mm-thick fused silica window to a probe table outside the vacuum target chamber. The diameter and energy were adjusted using an iris and ND filters to about $8 \mathrm{~mm}$ and $1 \mathrm{~mJ}$, respectively. A dispersive mirror array together with a variable-thickness glass wedge pair compensated the group delay dispersion accumulated during prefiber propagation and therefore ensured effective SPM inside the argon-filled hollow-core fiber with an inner diameter of $240 \mu \mathrm{m}$ and a length of $0.9 \mathrm{~m}$. With an argon pressure of 500 mbar, about $400 \mu \mathrm{J}$ were transmitted though the fiber. Thereafter, a second array of dispersive mirrors and a wedge pair were used to compress the pulse close to its Fourier limit.

\section{Simulated shadowgrams}

Previous studies on few-cycle shadowgraphy have used 3D-Cartesian PIC simulations with a separately initialized probe beam to simulate shadowgrams [70]. However, this approach becomes impractical for the large simulation windows as required in our case. Instead, we calculate a qualitative approximation of the shadowgrams of the simulated ion channels from quasi-3D simulation data in postprocessing. Using the dispersion relation of a cold plasma, we use the electron distribution to calculate the phase shift of a plane monochromatic wave traveling perpendicularly through the moving plasma in a static approximation. The electron distribution of the radially symmetric simulation is mapped onto a $3 \mathrm{D}$ grid where $\vec{e}_{z}$ is the direction of propagation of the driver and $\vec{e}_{y}$ the direction of propagation of the probe. Each layer in the $\vec{e}_{x \mid y}$ plane is shifted by $c \Delta y$ in the $\vec{e}_{z}$ direction, such that the distribution appears as moving with the speed of light as the probe propagates through it.

While our results show good agreement with the shadowgrams observed in experiment, it should be noted that there are a few limitations to our approach. First, it is only valid if the plasma wave does not evolve significantly while the probe transverses it. This is usually the case in wakefield acceleration and for all situations treated in this study, but special cases such as wave evolution in density gradients would be an exception. Here, one would need to use simulation data from different time steps. Furthermore, the cold plasma approximation is strictly valid only behind the plasma wave. Within the plasma wave the diffraction can be overestimated due to the reduced refractive index of relativistic electrons. If needed, this could be solved by analyzing not only density maps, but also the test particle data including their momenta.

[1] M. Tigner, Does Accelerator-Based Particle Physics Have a Future?, Phys. Today 54, No. 1, 36 (2001).

[2] C. J. Joshi and T. Katsouleas, Plasma Accelerators at the Energy Frontier and on Tabletops, Phys. Today 56, No. 6, 47 (2003).

[3] E. Esarey, P. Sprangle, J. Krall, and A. Ting, Overview of Plasma-Based Accelerator Concepts, IEEE Trans. Plasma Sci. 24, 252 (1996).

[4] P. Chen, J. M. Dawson, R. W. Huff, and T. Katsouleas, Acceleration of Electrons by the Interaction of a Bunched Electron Beam with a Plasma, Phys. Rev. Lett. 54, 693 (1985).

[5] P. Chen, J. J. Su, J. M. Dawson, K. L. F. Bane, and P. B. Wilson, Energy Transfer in the Plasma Wake-Field Accelerator, Phys. Rev. Lett. 56, 1252 (1986).

[6] J. B. Rosenzweig, D. B. Cline, B. Cole, H. Figueroa, W. Gai, R. Konecny, J. Norem, P. Schoessow, and J. Simpson, Experimental Observation of Plasma Wake-Field Acceleration, Phys. Rev. Lett. 61, 98 (1988).

[7] J. B. Rosenzweig, P. Schoessow, B. Cole, C. Ho, W. Gai, R. Konecny, S. Mtingwa, J. Norem, M. Rosing, and J. Simpson, Demonstration of Electron Beam Self-Focusing in Plasma Wake Fields, Phys. Fluids B 2, 1376 (1990).

[8] I. Blumenfeld, C. E. Clayton, F. J. Decker, M. J. Hogan, C. K. Huang, R. Ischebeck, R. Iverson, C. Joshi, T. Katsouleas, N. Kirby, W. Lu, K. A. Marsh, W. B. Mori, P. Muggli, E. Oz, R. H. Siemann, D. Walz, and M. M. Zhou, Energy Doubling of $42 \mathrm{GeV}$ Electrons in a Metre-Scale Plasma Wakefield Accelerator, Nature (London) 445, 741 (2007).

[9] M. Litos et al., High-Efficiency Acceleration of an Electron Beam in a Plasma Wakefield Accelerator, Nature (London) 515, 92 (2014). 
[10] S. Corde et al., Multi-Gigaelectronvolt Acceleration of Positrons in a Self-Loaded Plasma Wakefield, Nature (London) 524, 442 (2015).

[11] E. Adli et al., Acceleration of Electrons in the Plasma Wakefield of a Proton Bunch, Nature (London) 561, 363 (2018).

[12] B. Hidding, G. Pretzler, J. B. Rosenzweig, T. Königstein, D. Schiller, and D. L. Bruhwiler, Ultracold Electron Bunch Generation via Plasma Photocathode Emission and Acceleration in a Beam-Driven Plasma Blowout, Phys. Rev. Lett. 108, 035001 (2012).

[13] F. Li, J. F. Hua, X. L. Xu, C. J. Zhang, L. X. Yan, Y. C. Du, W. H. Huang, H. B. Chen, C.X. Tang, W. Lu, C. Joshi, W. B. Mori, and Y.Q. Gu, Generating High-Brightness Electron Beams via Ionization Injection by Transverse Colliding Lasers in a Plasma-Wakefield Accelerator, Phys. Rev. Lett. 111, 015003 (2013).

[14] A. Martinez de la Ossa, J. Grebenyuk, T. Mehrling, L. Schaper, and J. Osterhoff, High-Quality Electron Beams from Beam-Driven Plasma Accelerators by WakefieldInduced Ionization Injection, Phys. Rev. Lett. 111, 245003 (2013).

[15] A. Martinez de la Ossa, T. J. Mehrling, L. Schaper, M. J. V. Streeter, and J. Osterhoff, Wakefield-Induced Ionization Injection in Beam-Driven Plasma Accelerators, Phys. Plasmas 22, 093107 (2015).

[16] G. G. Manahan, A. F. Habib, P. Scherkl, P. Delinikolas, A. Beaton, A. Knetsch, O. Karger, G. Wittig, T. Heinemann, Z. M. Sheng, J. R. Cary, D. L. Bruhwiler, J. B. Rosenzweig, and B. Hidding, Single-Stage Plasma-Based Correlated Energy Spread Compensation for Ultrahigh $6 D$ Brightness Electron Beams, Nat. Commun. 8, 15705 (2017).

[17] A. R. Maier, A. Meseck, S. Reiche, C. B. Schroeder, T. Seggebrock, and F. Grüner, Demonstration Scheme for a Laser-Plasma-Driven Free-Electron Laser, Phys. Rev. X 2 , 031019 (2012).

[18] A. Caldwell, K. Lotov, A. Pukhov, and F. Simon, ProtonDriven Plasma-Wakefield Acceleration, Nat. Phys. 5, 363 (2009).

[19] E. Esarey, C. B. Schroeder, and W. P. Leemans, Physics of Laser-Driven Plasma-Based Electron Accelerators, Rev. Mod. Phys. 81, 1229 (2009).

[20] C. Joshi, The Development of Laser- and Beam-Driven Plasma Accelerators as an Experimental Field, Phys. Plasmas 14, 055501 (2007).

[21] M. J. Hogan, T. O. Raubenheimer, A. Seryi, P. Muggli, T. Katsouleas, C. Huang, W. Lu, W. An, K. A. Marsh, W. B. Mori, C.E. Clayton, and C. Joshi, Plasma Wakefield Acceleration Experiments at FACET, New J. Phys. 12, 055030 (2010).

[22] J.-L. Vay and R. Lehe, Simulations for Plasma and Laser Acceleration, Rev. Accel. Sci. Techol. 09, 165 (2016).

[23] V. Malka, J. Faure, Y. A. Gauduel, E. Lefebvre, A. Rousse, and K. T. Phuoc, Principles and Applications of Compact Laser-Plasma Accelerators, Nat. Phys. 4, 447 (2008).

[24] S. M. Hooker, Developments in Laser-Driven Plasma Accelerators, Nat. Photonics 7, 775 (2013).

[25] B. Hidding, T. Königstein, J. Osterholz, S. Karsch, O. Willi, and G. Pretzler, Monoenergetic Energy Doubling in a
Hybrid Laser-Plasma Wakefield Accelerator, Phys. Rev. Lett. 104, 195002 (2010).

[26] K. H. Pae, I. W. Choi, and J. Lee, Self-Mode-Transition from Laser Wakefield Accelerator to Plasma Wakefield Accelerator of Laser-Driven Plasma-Based Electron Acceleration, Phys. Plasmas 17, 123104 (2010).

[27] O. Lundh, J. Lim, C. Rechatin, L. Ammoura, A. Ben-Ismail, X. Davoine, G. Gallot, J. P. Goddet, E. Lefebvre, V. Malka, and J. Faure, Few Femtosecond, Few Kiloampere Electron Bunch Produced by a Laser-Plasma Accelerator, Nat. Phys. 7, 219 (2011).

[28] C. Joshi, E. Adli, W. An, C.E. Clayton, S. Corde, S. Gessner, M. J. Hogan, M. Litos, W. Lu, K. A. Marsh, W. B. Mori, N. Vafaei-Najafabadi, B. O'Shea, X. Xu, G. White, and V. Yakimenko, Plasma Wakefield Acceleration Experiments at FACET II, Plasma Phys. Controlled Fusion 60, 034001 (2018).

[29] A. Aschikhin et al., The FLASHForward Facility at DESY, Nucl. Instrum. Methods Phys. Res., Sect. A 806, 175 (2016).

[30] E. Gschwendtner et al., AWAKE, The Advanced Proton Driven Plasma Wakefield Acceleration Experiment at CERN, Nucl. Inst. Methods Phys. Res., Sect, A 829, 76 (2016).

[31] M. B. Schwab, A. Savert, O. Jackel, J. Polz, M. Schnell, T. Rinck, L. Veisz, M. Möller, P. Hansinger, G. G. Paulus, and M. C. Kaluza, Few-Cycle Optical Probe-Pulse for Investigation of Relativistic Laser-Plasma Interactions, Appl. Phys. Lett. 103, 191118 (2013).

[32] M.C. Downer, R. Zgadzaj, A. Debus, U. Schramm, and M. C. Kaluza, Diagnostics for Plasma-Based Electron Accelerators, Rev. Mod. Phys. 90, 035002 (2018).

[33] With a central wavelength $\lambda_{1}=800 \mathrm{~nm}$ the contrast of plasma waves in the shadowgram at densities below $10^{18} \mathrm{~cm}^{-3}$ is poor, due to the scaling of the refractive index $\eta=\sqrt{1-\lambda_{1}^{2} / \lambda_{p}^{2}}$. Lowering the density $\left(n_{0} \propto \lambda_{p}^{-2}\right)$ even further, e.g., by only 2 orders of magnitude to $10^{16} \mathrm{~cm}^{-3}$ the wavelength of the probe needs to be scaled accordingly by one order of magnitude to about $8 \mu \mathrm{m}$, which is challenging on both laser and detector side.

[34] J. Wenz, A. Döpp, K. Khrennikov, S. Schindler, M. F. Gilljohann, H. Ding, J. Götzfried, A. Buck, J. Xu, M. Heigoldt, W. Helml, L. Veisz, and S. Karsch, Dual-Energy Electron Beams from a Compact Laser-Driven Accelerator, Nat. Photon. (in press).

[35] J. Vieira, W. B. Mori, and P. Muggli, Hosing Instability Suppression in Self-Modulated Plasma Wakefields, Phys. Rev. Lett. 112, 205001 (2014).

[36] P. E. Masson-Laborde, M. Z. Mo, A. Ali, S. Fourmaux, P. Lassonde, J. C. Kieffer, W. Rozmus, D. Teychenne, and R. Fedosejevs, Giga-Electronvolt Electrons due to a Transition from Laser Wakefield Acceleration to Plasma Wakefield Acceleration, Phys. Plasmas 21, 123113 (2014).

[37] E. Guillaume, A. Döpp, C. Thaury, A. Lifschitz, J. P. Goddet, A. Tafzi, F. Sylla, G. Iaquanello, T. Lefrou, P. Rousseau, K. Ta Phuoc, and V. Malka, Physics of FullyLoaded Laser-Plasma Accelerators, Phys. Rev. ST Accel. Beams 18, 061301 (2015). 
[38] M. Heigoldt, A. Popp, K. Khrennikov, J. Wenz, S. W. Chou, S. Karsch, S. I. Bajlekov, S. M. Hooker, and B. Schmidt, Temporal Evolution of Longitudinal Bunch Profile in a Laser Wakefield Accelerator, Phys. Rev. ST Accel. Beams 18, 121302 (2015).

[39] S. Corde, C. Thaury, K. Ta Phuoc, A. Lifschitz, G. Lambert, J. Faure, O. Lundh, E. Benveniste, A. Ben-Ismail, L. Arantchuk, A. Marciniak, A. Stordeur, P. Brijesh, A. Rousse, A. Specka, and V. Malka, Mapping the X-Ray Emission Region in a Laser-Plasma Accelerator, Phys. Rev. Lett. 107, 215004 (2011).

[40] S. Chou, J. Xu, K. Khrennikov, D. E. Cardenas, J. Wenz, M. Heigoldt, L. Hofmann, L. Veisz, and S. Karsch, Colches, Phys. Rev. Lett. 117, 144801 (2016).

[41] S. Kuschel, D. Hollatz, T. Heinemann, O. Karger, M. B. Schwab, D. Ullmann, A. Knetsch, A. Seidel, C. Rödel, M. Yeung, M. Leier, A. Blinne, H. Ding, T. Kurz, D. J. Corvan, A. Savert, S. Karsch, M. C. Kaluza, B. Hidding, and M. Zepf, Demonstration of Passive Plasma Lensing of a Laser Wakefield Accelerated Electron Bunch, Phys. Rev. ST Accel. Beams 19, 071301 (2016).

[42] K. Khrennikov, J. Wenz, A. Buck, J. Xu, M. Heigoldt, L. Veisz, and S. Karsch, Tunable All-Optical Quasimonochromatic Thomson X-Ray Source in the Nonlinear Regime, Phys. Rev. Lett. 114, 195003 (2015).

[43] K. Schmid, A. Buck, C. M. S. Sears, J. M. Mikhailova, R. Tautz, D. Herrmann, M. Geissler, F. Krausz, and L. Veisz, Density-Transition Based Electron Injector for Laser Driven Wakefield Accelerators, Phys. Rev. ST Accel. Beams 13, 091301 (2010).

[44] A. Buck, J. Wenz, J. Xu, K. Khrennikov, K. Schmid, M. Heigoldt, J. M. Mikhailova, M. Geissler, B. Shen, F. Krausz, S. Karsch, and L. Veisz, Shock-Front Injector for HighQuality Laser-Plasma Acceleration, Phys. Rev. Lett. 110, 185006 (2013).

[45] E. Guillaume, A. Döpp, C. Thaury, K. Ta Phuoc, A. Lifschitz, G. Grittani, J. P. Goddet, A. Tafzi, S. W. Chou, L. Veisz, and V. Malka, Electron Rephasing in a LaserWakefield Accelerator, Phys. Rev. Lett. 115, 155002 (2015).

[46] T. Kurz, J. P. Couperus, J. M. Krämer, H. Ding, S. Kuschel, A. Köhler, O. Zarini, D. Hollatz, D. Schinkel, R. D’Arcy, J.-P. Schwinkendorf, J. Osterhoff, A. Irman, U. Schramm, and S. Karsch, Calibration and Cross-Laboratory Implementation of Scintillating Screens for Electron Bunch Charge Determination, Rev. Sci. Instrum. 89, 093303 (2018).

[47] See Supplemental Material at http://link.aps.org/ supplemental/10.1103/PhysRevX.9.011046 for a discussion on differences between PWFA and LWFA, full-view shadowgrams of Figs. 2 and 3, close-ups of the drivers in Fig. 3, and a larger plot of the ion distribution with transverse electric fields of the simulation in Fig. 4, which includes Ref. [48].

[48] A. G. R. Thomas, S. P. D. Mangles, Z. Najmudin, M. C. Kaluza, C. D. Murphy, and K. Krushelnick, Measurements of Wave-Breaking Radiation from a Laser-Wakefield Accelerator, Phys. Rev. Lett. 98, 054802 (2007).

[49] C. Thaury, E. Guillaume, A. Döpp, R. Lehe, A. Lifschitz, K. Ta Phuoc, J. Gautier, J. P. Goddet, A. Tafzi, A. Flacco, F. Tissandier, S. Sebban, A. Rousse, and V. Malka,
Demonstration of Relativistic Electron Beam Focusing by a Laser-Plasma Lens, Nat. Commun. 6, 6860 (2015).

[50] M. Tzoufras, W. Lu, F. S. Tsung, C. Huang, W. B. Mori, T. Katsouleas, J. Vieira, R. A. Fonseca, and L. O. Silva, Beam Loading in the Nonlinear Regime of Plasma-Based Acceleration, Phys. Rev. Lett. 101, 145002 (2008).

[51] A. Popp, J. Vieira, J. Osterhoff, Z. Major, R. Horlein, M. Fuchs, R. Weingartner, T. P. Rowlands-Rees, M. Marti, R. A. Fonseca, S. F. Martins, L. O. Silva, S. M. Hooker, F. Krausz, F. Grüner, and S. Karsch, All-Optical Steering of Laser-Wakefield-Accelerated Electron Beams, Phys. Rev. Lett. 105, 215001 (2010).

[52] C. Thaury, F. Quere, J. P. Geindre, A. Levy, T. Ceccotti, P. Monot, M. Bougeard, F. Reau, P. D'Oliveira, P. Audebert, R. Marjoribanks, and P. H. Martin, Plasma Mirrors For Ultrahigh-Intensity Optics, Nat. Phys. 3, 424 (2007).

[53] B. H. Shaw, S. Steinke, J. van Tilborg, and W. P. Leemans, Reflectance Characterization of Tape-Based Plasma Mirrors, Phys. Plasmas 23, 063118 (2016).

[54] D. L. Bruhwiler, D. A. Dimitrov, J. R. Cary, E. Esarey, W. Leemans, and R. E. Giacone, Particle-in-Cell Simulations of Tunneling Ionization Effects in Plasma-Based Accelerators, Phys. Plasmas 10, 2022 (2003).

[55] R. A. Fonsecaet al., OSIRIS: A Three-Dimensional, Fully Relativistic Particle in Cell Code for Modeling Plasma Based Accelerators, in Computational Science-ICCS 2002. ICCS 2002, edited by P. M. A. Sloot, A. G. Hoekstra, C. J. K. Tan, and J. J. Dongarra, Lecture Notes in Computer Science Vol. 2331 (Springer, Berlin, Heidelberg, 2002)

[56] S. P. D. Mangles, B. R. Walton, M. Tzoufras, Z. Najmudin, R. J. Clarke, A. E. Dangor, R. G. Evans, S. Fritzler, A. Gopal, C. Hernandez-Gomez, W. B. Mori, W. Rozmus, M. Tatarakis, A. G. R. Thomas, F. S. Tsung, M. S. Wei, and K. Krushelnick, Electron Acceleration in Cavitated Channels Formed by a Petawatt Laser in Low-Density Plasma, Phys. Rev. Lett. 94, 245001 (2005).

[57] R. Tarkeshian, J. L. Vay, R. Lehe, C. B. Schroeder, E. H. Esarey, T. Feurer, and W. P. Leemans, Transverse SpaceCharge Field-Induced Plasma Dynamics for Ultraintense Electron-Beam Characterization, Phys. Rev. X 8, 021039 (2018).

[58] The derivation of this formula is analogous to ponderomotive motion in a laser field as outlined in common text books, e.g., Ref. [59].

[59] A. Macchi, A Superintense Laser-Plasma Interaction Theory Primer, Springer Briefs in Physics (Springer, New York, 2013).

[60] L. M. Gorbunov, P. Mora, and A. A. Solodov, Plasma Ions Dynamics in the Wake of a Short Laser Pulse, Phys. Rev. Lett. 86, 3332 (2001).

[61] L. M. Gorbunov, P. Mora, and A. A. Solodov, Dynamics of a Plasma Channel Created by the Wakefield of a Short Laser Pulse, Phys. Plasmas 10, 1124 (2003).

[62] J. B. Rosenzweig, A. M. Cook, A. Scott, M. C. Thompson, and R. B. Yoder, Effects of Ion Motion in Intense BeamDriven Plasma Wakefield Accelerators, Phys. Rev. Lett. 95, 195002 (2005).

[63] J. Vieira, R. A. Fonseca, W. B. Mori, and L. O. Silva, Ion Motion in Self-Modulated Plasma Wakefield Accelerators, Phys. Rev. Lett. 109, 145005 (2012). 
[64] J. Vieira, R. A. Fonseca, W. B. Mori, and L. O. Silva, Ion Motion in the Wake Driven by Long Particle Bunches in Plasmas, Phys. Plasmas 21, 056705 (2014).

[65] R. Keinigs and M. E. Jones, Two-Dimensional Dynamics of the Plasma Wakefield Accelerator, Phys. Fluids 30, 252 (1987).

[66] M. Downer (private communication). Because of the use of much lower plasma density $\left(5 \times 10^{16} \mathrm{~cm}^{-3}\right)$, the feature appears at later times in this experiment.

[67] X. Wang et al., Quasi-Monoenergetic Laser-Plasma Acceleration of Electrons to $2 \mathrm{GeV}$, Nat. Commun. 4, 1988 (2013).

[68] J. P. Couperus, R. Pausch, A. Köhler, O. Zarini, J. M. Krämer, M. Garten, A. Huebl, R. Gebhardt, U. Helbig,
S. Bock, K. Zeil, A. Debus, M. Bussmann, U. Schramm, and A. Irman, Demonstration of a Beam Loaded Nanocoulomb-Class Laser Wakefield Accelerator, Nat. Commun. 8, 487 (2017).

[69] J. Ferri, S. Corde, A. Döpp, A. Lifschitz, A. Doche, C. Thaury, K. Ta Phuoc, B. Mahieu, I. A. Andriyash, V. Malka, and X. Davoine, High-Brilliance Betatron-Ray Source Powered by Laser-Accelerated Electrons, Phys. Rev. Lett. 120, 254802 (2018).

[70] E. Siminos, S. Skupin, A. Savert, J. M. Cole, S. P. D. Mangles, and M. C. Kaluza, Modeling Ultrafast Shadowgraphy in Laser-Plasma Interaction Experiments, Plasma Phys. Controlled Fusion 58, 065004 (2016). 